\title{
To sacrifice or replace the posterior cruciate ligament in primary total knee arthroplasty?
}

\author{
O. Şahap Atik, $M D^{1}\left(\mathbb{D}\right.$, Sualp Turan, $M D^{2}$ (D) \\ ${ }^{1}$ Professor of Orthopedic Surgery, Turkish Joint Diseases Foundation, Ankara, Turkey \\ ${ }^{2}$ Department of Orthopedics and Traumatology, Health Sciences University, Bilkent City Hospital, Ankara, Turkey
}

Treatment options for osteoarthritis of the knee joint include high tibial osteotomy, unicompartmental knee arthroplasty, and total knee arthroplasty (TKA). ${ }^{[1-3]}$

Both cruciate-retaining (CR) and posteriorstabilized (PS) implants are commonly used for primary TKA. However, there has been continuing debate about whether to sacrifice or replace the posterior cruciate ligament when performing TKA. The superiority of CR-TKA versus PS-TKA for obtaining knee joint stability with functional improvement is still controversial.

A meta-analysis of eight randomized controlled trials (RCTs) involving 888 patients revealed that CR- and PS-TKA have similar clinical outcomes with regard to knee function, postoperative knee pain and other complications. Implant survivorship for both posterior CR- and PS-TKA is satisfactory, with no differences between them at short- and middle-term follow-up. ${ }^{[4]}$

In another meta-analysis of 36 clinical trials with levels of evidence of I and II, involving 4,052

Received: June 13, 2020

Accepted: August 12, 2020

Published online: September 11, 2020

Correspondence: $O$. Şahap Atik, MD. Turkish Joint Diseases

Foundation, Mustafa Kemal Mah., Dumlupınar Bul., 274/2, C2 Blok,

Ofis 5, 06900 Çankaya, Ankara, Türkiye.

E-mail: satikmd@gmail.com

Doi: 10.5606/ehc. 2020.57897

Citation: Atik OS, Turan S. To sacrifice or replace the posterior cruciate ligament in primary total knee arthroplasty? Jt Dis Relat surg 2020;31(3):656-657.

(02020 All right reserved by the Turkish Joint Diseases Foundation

This is an open access article under the terms of the Creative Commons Attribution-NonCommercial License, which permits use, distribution and reproduction in any medium, provided the original work is properly cited and is not used for commercial purposes (http://creativecommons.org/licenses/by-nc/4.0/). patients, no differences were reported for two groups regarding anterior knee pain, knee joint instability or revision rate. ${ }^{[5]}$

In a case-control study of 268 patients who underwent CR-TKA versus 211 PS-TKAs, with the same arthroplasty system, and a minimum follow-up of 10 years, the superiority of one design over the other was not found. Both designs can be used expecting long-term successful outcomes and high survival rates. ${ }^{[6]}$

A meta-analysis of 1,114 patients revealed a significant difference in statistical analysis of flexion and range of motion (ROM) in favor of PS knees, while no difference was detected in complication rates. The clinical importance of this finding remains unknown. ${ }^{[7]}$

A total of 42 knees were investigated, with equal representation in the PS- and CR-TKA groups, showing kinematic and functional differences that favored PS-TKA. The results suggest posterior cruciate ligament insufficiency in CR-TKA, indicating that the cam-post systems in PS-TKA may better maintain knee kinematics and function at long term. ${ }^{[8]}$

In a RCT, both PS- and CR-TKA performed well with patients reporting acceptable levels of healthrelated quality of life in up to 10 years postoperatively. Low levels of revision or reoperation were reported in both groups. ${ }^{[9]}$

A matched paired study comparing in a matched cohort, there were no significant differences in functional score, overall ROM or patient satisfaction between PS- and CR-TKA at 10-year follow-up. However, PS knees had a greater score improvement in ROM compared with CR knees. ${ }^{[10]}$

The influence of posterior tibial slope was investigated in a study. Eight degrees or more posterior tibial slope in CR-TKA using prosthetics designed with 
high geometric conformity to the medial articular surface did not affect the anteroposterior position and external rotation, but increased the postoperative maximum flexion angle and ROM. ${ }^{[11]}$

In conclusion, both prosthetic implants provide a feasible solution to treat osteoarthritis of the knee joint. The PS-TKA reported improvements in the knee ROM. No clinically relevant significant differences concerning the analyzed scores were evidenced. Furthermore, no statistically significant relevant differences in complications were detected. Therefore, strict adherence to surgical indications, the status of the posterior cruciate ligament and understanding the differences in surgical principles may be more important than the selection of a CR or PS prosthesis. ${ }^{[12]}$

\section{Declaration of conflicting interests}

The authors declared no conflicts of interest with respect to the authorship and/or publication of this article.

\section{Funding}

The authors received no financial support for the research and/or authorship of this article.

\section{REFERENCES}

1. Papp M, Zsákai Z, Gömöri A. Comparison of total knee arthroplasty after combined high tibial osteotomy with a matched group of primary total knee arthroplasty. Eklem Hastalik Cerrahisi 2019;30:79-84.

2. Sever GB, Cankuş C. The long-term results of cemented Oxford unicompartmental knee arthroplasty: A singlecenter experience. Eklem Hastalik Cerrahisi 2019;30:233-40.

3. Lacko M, Jarčuška P, Schreierova D, Lacková A, Gharaibeh A. Tranexamic acid decreases the risk of revision for acute and delayed periprosthetic joint infection after total knee replacement. Jt Dis Relat Surg 2020;31:8-13.

4. Li N, Tan Y, Deng Y, Chen L. Posterior cruciate-retaining versus posterior stabilized total knee arthroplasty: a metaanalysis of randomized controlled trials. Knee Surg Sports Traumatol Arthrosc 2014;22:556-64.

5. Migliorini F, Eschweiler J, Tingart M, Rath B. Posteriorstabilized versus cruciate-retained implants for total knee arthroplasty: a meta-analysis of clinical trials. Eur J Orthop Surg Traumatol 2019;29:937-46.

6. Serna-Berna R, Lizaur-Utrilla A, Vizcaya-Moreno MF, Miralles Muñoz FA, Gonzalez-Navarro B, Lopez-Prats FA. Cruciate-retaining vs posterior-stabilized primary total arthroplasty. Clinical outcome comparison with a minimum follow-up of 10 years. J Arthroplasty 2018;33:2491-5.

7. Bercik MJ, Joshi A, Parvizi J. Posterior cruciate-retaining versus posterior-stabilized total knee arthroplasty: a metaanalysis. J Arthroplasty 2013;28:439-44.

8. Broberg JS, Ndoja S, MacDonald SJ, Lanting BA, Teeter MG. Comparison of contact kinematics in posterior-stabilized and cruciate-retaining total knee arthroplasty at long-term follow-up. J Arthroplasty 2020;35:272-7.

9. Beaupre LA, Sharifi B, Johnston DWC. A randomized clinical trial comparing posterior cruciate-stabilizing vs posterior cruciate-retaining prostheses in primary total knee arthroplasty: 10-year follow-up. J Arthroplasty 2017;32:818-23.

10. Mayne A, Harshavardhan HP, Johnston LR, Wang W, Jariwala A. Cruciate Retaining compared with Posterior Stabilised Nexgen total knee arthroplasty: results at 10 years in a matched cohort. Ann R Coll Surg Engl 2017;99:602-6.

11. Fujito T, Tomita $T$, Yamazaki $T$, Oda K, Yoshikawa $\mathrm{H}$, Sugamoto K. Influence of posterior tibial slope on kinematics after cruciate-retaining total knee arthroplasty. J Arthroplasty 2018;33:3778-82.e1.

12. Atik OS. Is there something new and interesting in my article? Eklem Hastalik Cerrahisi 2019;30:69. 